\title{
Article \\ Deletion of the Spata3 Gene Induces Sperm Alterations and In Vitro Hypofertility in Mice
}

\author{
Marie-Sophie Girault ${ }^{1, \dagger}$, Sophie Dupuis ${ }^{1, \dagger}{ }^{+}$, Côme Ialy-Radio ${ }^{1}$, Laurence Stouvenel ${ }^{1}$, Cécile Viollet ${ }^{1}$, \\ Rémi Pierre ${ }^{1}$, Maryline Favier ${ }^{1}$, Ahmed Ziyyat ${ }^{1,2,+}+\mathbb{D}$ and Sandrine Barbaux ${ }^{1, *,+}$ \\ 1 Institut Cochin, Université de Paris, INSERM, CNRS, F-75014 Paris, France; \\ marie-sophie.girault@inserm.fr (M.-S.G.); sophie.dupuis@inserm.fr (S.D.); come.ialy-radio@inserm.fr (C.I.-R.); \\ laurence.stouvenel@inserm.fr (L.S.); violletcecile@gmail.com (C.V.); remi.pierre@inserm.fr (R.P.); \\ maryline.favier@inserm.fr (M.F.); aziyyat@yahoo.fr (A.Z.) \\ 2 Service d'Histologie, d'Embryologie, Biologie de la Reproduction, AP-HP, Hôpital Cochin, \\ F-75014 Paris, France \\ * Correspondence: sandrine.barbaux@inserm.fr \\ + These authors contributed equally to this work. M.-S.G. and S.D. are co-first authors; A.Z. and S.B. are \\ co-last authors.
}

Citation: Girault, M.-S.; Dupuis, S.; Ialy-Radio, C.; Stouvenel, L.; Viollet, C.; Pierre, R.; Favier, M.; Ziyyat, A.; Barbaux, S. Deletion of the Spata3 Gene Induces Sperm Alterations and In Vitro Hypofertility in Mice. Int. J. Mol. Sci. 2021, 22, 1959. https:// doi.org/10.3390/ijms22041959

Academic Editor: Donato Gemmati Received: 15 January 2021

Accepted: 12 February 2021

Published: 16 February 2021

Publisher's Note: MDPI stays neutra with regard to jurisdictional claims in published maps and institutional affiliations.

Copyright: (C) 2021 by the authors Licensee MDPI, Basel, Switzerland. This article is an open access article distributed under the terms and conditions of the Creative Commons Attribution (CC BY) license (https:// creativecommons.org/licenses/by/ $4.0 /)$.

\begin{abstract}
Thanks to the analysis of an Interspecific Recombinant Congenic Strain (IRCS), we previously defined the Mafq1 quantitative trait locus as an interval on mouse Chromosome 1 associated with male hypofertility and ultrastructural abnormalities. We identified the Spermatogenesis associated protein 3 gene (Spata3 or Tsarg1) as a pertinent candidate within the Mafq1 locus and performed the CRISPR-Cas9 mediated complete deletion of the gene to investigate its function. Male mice deleted for Spata3 were normally fertile in vivo but exhibited a drastic reduction of efficiency in in vitro fertilization assays. Mobility parameters were normal but ultrastructural analyses revealed acrosome defects and an overabundance of lipids droplets in cytoplasmic remnants. The deletion of the Spata3 gene reproduces therefore partially the phenotype of the hypofertile IRCS strain.
\end{abstract}

Keywords: Spata3; infertility; acrosome; spermatogenesis; sperm; mouse model; lipid droplets

\section{Introduction}

Genes necessary for testis function, particularly in post-meiotic germ cells, are extremely numerous [1]. However, the function and character of dispensability of these genes is only partially known. Their responsibility in clinical cases of human male infertility is therefore also only partially deciphered.

For example, globozoospermia, a very rare form of male infertility characterized by round-shaped sperm heads that present a complete lack or an abnormal setup of their acrosome, can be explained by alterations of the DPY19L2 gene in about $60 \%$ of reported cases [2,3]. Some rare case reports have also revealed mutations of the SPATA16, ZPBP1 and PICK1 genes [4-6]. But the defect responsible for some remaining clinical cases is not yet identified and the biogenesis of the acrosome is still not completely elucidated. Animal models can help with suggesting some other genes involved in the process.

Recently, we reported the analysis of a mouse model exhibiting a phenotype of spermatogenesis alterations including anomalies of the acrosome. We identified in an Interspecific Recombinant Congenic Strain (IRCS) a region of mouse chromosome 1 whose presence in a Mus spretus version within a Mus musculus domesticus (C57Bl/6) background is associated with signs reminiscent of globozoospermia. This quantitative trait locus (QTL), named Mafq1 for male fertility QTL chromosome 1, is associated with an interval of approximately $4 \mathrm{Mb}$ between markers D1Mit438 and D1Mit305 [7].

This specific interval contains more than 70 coding genes. Among them, we concentrated our research on the Spata3 gene (Spermatogenesis associated protein 3, also known 
as Testis and Spermatogenesis cell Apoptosis Related Gene 1 or Tsarg1) [8,9]. We consider it as one of the best candidates to explain the hypofertility phenotype of the Rc3 strain, on the basis of various arguments.

Spata3 is expressed specifically in the testis both in mice and humans. Precisely, its expression can be detected during spermatogenesis from the round spermatid stage to mature sperm [7]. Spata3 is conserved in many mammalian species, with mouse and human proteins sharing $55 \%$ of their identity.

No data are available on the function of the SPATA3 protein, the SPATA family being a catalogue of proteins sharing an expression in the testis without any similarity of sequence neither function. Furthermore, no specific functional domain can be predicted from its sequence and no mouse model is available to determine its role. However, the human SPATA3 gene has been reported to be among the most down-regulated genes in the testis of infertile men diagnosed with nonobstructive azoospermia [10]. Rare variants of this gene were also associated with prostate cancer [11].

Comparison of the sequence of the Spata3 gene between the Mus spretus and the C57Bl/ 6 versions highlights many variations: 14 missense changes in the different isoforms and at least 16 UTR variants (Table S1). These variations could be responsible for important modifications of the structure and/or the function of the spretus SPATA3 protein compared to the $\mathrm{C} 57 \mathrm{Bl} / 6$ version. This spretus version of the protein could therefore be inapt to interact/bind/function with any receptor/cofactor/partner in the C57Bl/ 6 version in the IRCS model to accomplish its normal activity, resulting in the hypofertility phenotype observed in the Rc3 IRCS male mice [7].

We therefore decided to produce a mouse line invalidated for the Spata3 gene in order to decipher the function of this gene. Though male mice lacking Spata3 expression are fully fertile in vivo, their fertility in vitro is drastically affected and some ultrastructural anomalies can be observed in sperm samples.

\section{Results}

\subsection{Spata3 Is Expressed in Sperm and Localized on the Acrosome}

We used a specific antibody to detect SPATA3 in testis slides. SPATA3 is expressed from the round spermatid stage to mature sperm. It is specifically localized at the acrosome region (Figure 1a-d). No signal was obtained when secondary antibody was used alone (Figure 1e,f). We also confirmed its presence at the mRNA level by RT-PCR, as shown in Figure 2b. Quantitative single-cell RNA sequencing analyses confirm a germ-cell specific expression from the round spermatid stage in humans and mice (Reprogenomics Viewer) $[12,13]$.

\subsection{Spata3 Gene Deleted Male Mice Are Fertile In Vivo}

Mice invalidated for the Spata3 gene were generated by microinjecting embryos with RNA guides targeting upstream of the first coding exon (exon 1bis) and downstream of the last coding exon (exon 3) (Figure 2a). Two different though very similar lines encompassing an $8547 \mathrm{nt}$ deletion and an $8507 \mathrm{nt}$ deletion of the gene locus, respectively, were selected and maintained. Identification of animals presenting a deletion of Spata3 was obtained by PCR amplification and sequencing of the locus as illustrated in Figure 2c. Different crossings were performed to produce homozygous knock-out animals (KO). Females Spata3 KO were normally fertile. As the antibody previously used for immunofluorescence detection (Figure 1) is no longer commercialized and no other validated antibody is available for Western blotting, the absence of expression of the Spata3 gene could only be checked by RT-PCR. No amplification of Spata3 could be observed in the testis of KO males compared to wild-type (WT) samples whereas the cDNA of a testis-specific gene, Prm1, could be detected in both samples (Figure 2b) [14]. Spata3 KO males exhibited a normal sexual behavior and after mating with WT females, could sire offspring (Figure 3a). The average litter size was not different when pups were born from KO males $(7.1 \pm 0.9 ; n=9)$ or from WT males $(6.7 \pm 0.5 ; n=10, p=0.71)$. This result suggests that in vivo the Spata3 gene is 
dispensable for sperm function and fertility. Similar results were obtained with the two $\mathrm{KO}$ strains but only one representative strain is shown.
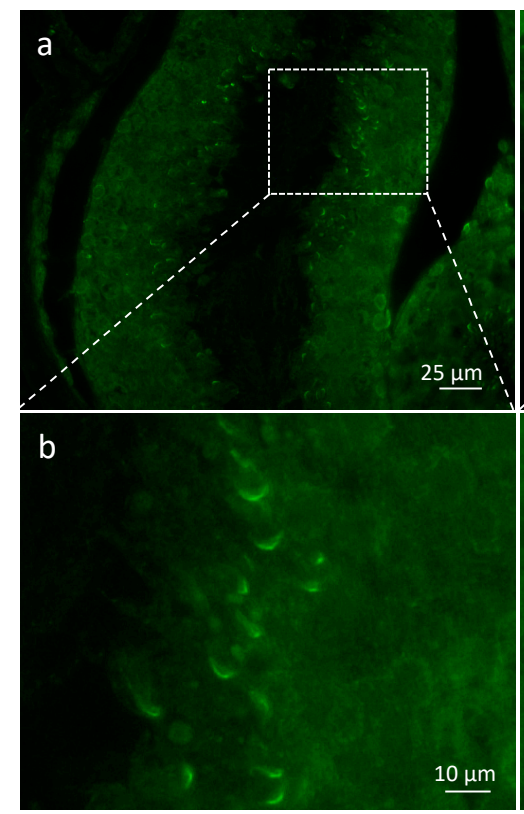

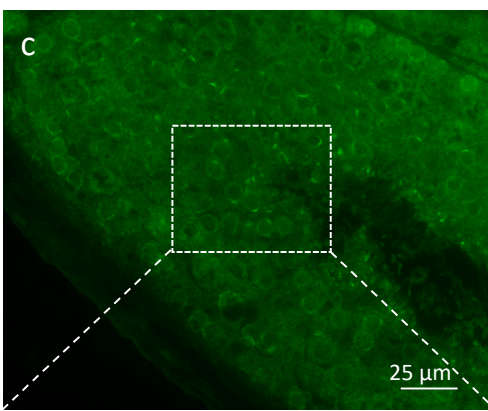

d

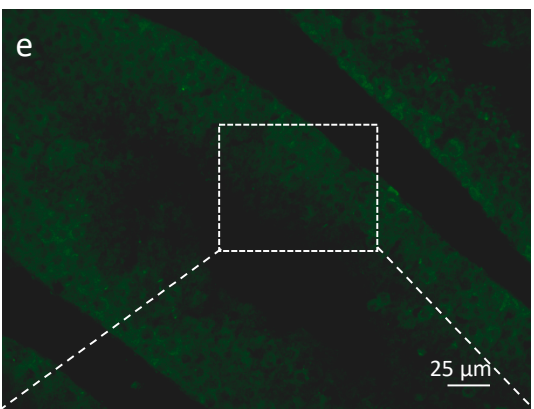

$f$

Figure 1. Presence of SPATA3 at the acrosome level. (a,c) Immunostaining for SPATA3 detection. (e) shows negative control using secondary antibody alone and $(\mathbf{b}, \mathbf{d}, \mathbf{f})$ show a higher magnification of regions framed in $(\mathbf{a}, \mathbf{c}, \mathbf{e})$, respectively.

\subsection{Spata3 Gene Deleted Male Mice Have a Reduced Fertility In Vitro}

In vitro fertilization assays were set up to further analyze the sperm quality. The fertilization rate (FR: number of fertilized oocytes among the total) of cumulus-intact WT oocytes was $34.9 \pm 3.9 \%(n=147)$ when WT sperm was used and $7.3 \pm 2.1 \%(n=150)$ when the sperm was obtained from KO males $(p<0.0001)$ (Figure $3 b)$. When zona-free oocytes from WT females were inseminated with sperm from WT or KO males, the fertilization index (FI: the average number of fused sperms per oocyte) was $0.92 \pm 0.06(n=108)$ and $0.35 \pm 0.04(n=118)$, respectively $(p<0.0001)$ (Figure $3 c)$. These observations confirm that Spata3 is not essential for in vivo fertilization but that in vitro, its absence affects the successful process of fertilization.

\subsection{Sperm from Spata3 Gene Deleted Male Mice Have Structural Anomalies}

In order to identify why sperm from Spata3 $\mathrm{KO}$ mice were not as efficient to fertilize in vitro, we decided to characterize it. Histology of the testes of $\mathrm{KO}$ males showed no abnormalities (Figure 4a). Observation of sperm under a light microscope failed to show any obvious defect (Figure $4 \mathrm{~b}$ ). Counts from epididymal sperm were not different between $\mathrm{WT}$ and $\mathrm{KO}$ males (Figure 4c). Similarly, spontaneous acrosome reaction occurred at the same level in WT and KO males after sperm capacitation (Figure 4d). Analyses using the Computer Aided Sperm Analysis (CASA) system did not reveal any difference between the control and $\mathrm{KO}$ sperm for any parameters, including curvilinear velocity (VCL), beat/cross frequency (BCF) and amplitude of lateral head displacement (ALH), as shown in Figure 4e,f. 


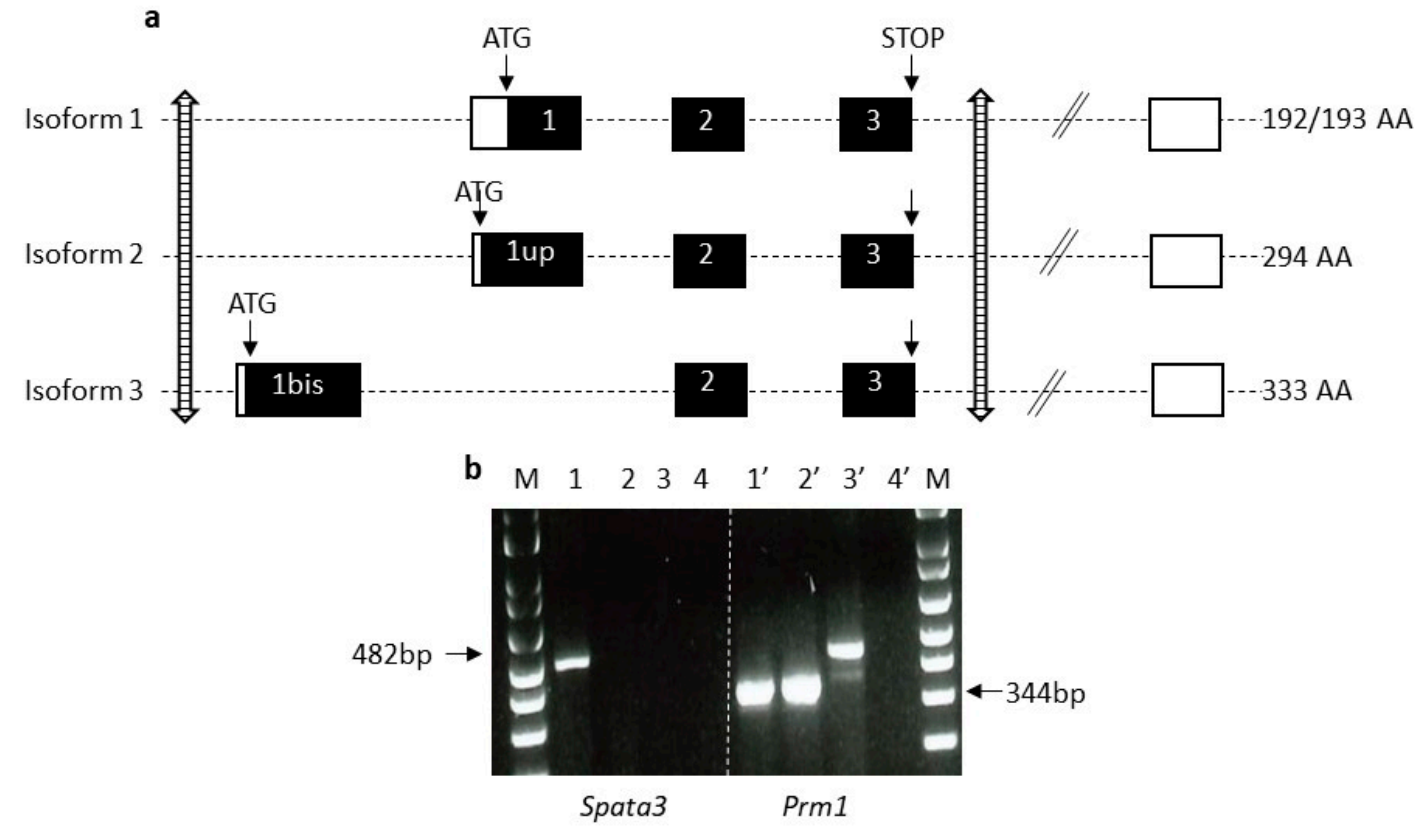

\section{C}

Upstream Exon1 of Spata3

GTCTTTCCTITGGCTAACAGGGTCCCAGGCCATTGGGACAAGAGAACCGGACAGACATGGA'ACAGAGGCCTGGGGAGCAGCCCTCAAGAGCCCCA

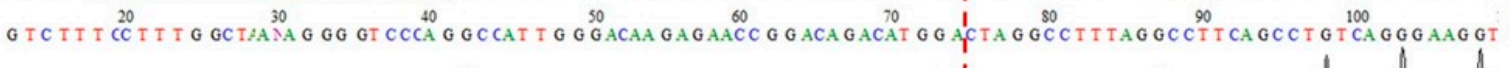

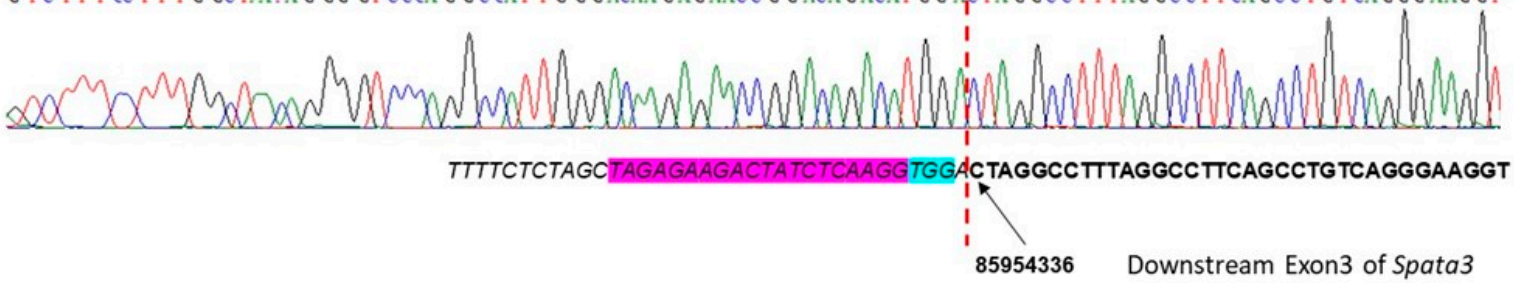

Figure 2. Strategy of deletion of the mouse Spata3 gene. (a). Map of the mouse Spata3 gene locus. The organization of the mouse Spata3 gene is represented with the main exons (coding in black boxes, non-coding in white boxes). Isoforms 1, 2 and 3 represent the major isoforms. The position of the sites targeted by RNA guides is marked by striped arrows. Genome version GRCm38.p6. (b). Expression of Spata3 and Prm1 genes in testis samples. 1 and 1', wild-type (WT) cDNA; 2 and 2', knock-out animals (KO) cDNA; 3 and $3^{\prime}$, WT genomic DNA; 4 and $4^{\prime}$, negative control; M, molecular weight marker. (c). Chromatogram of the deletion site including alignment with the WT sequence. The RNA guides are in purple and the PAM sites in blue. The positions of deletion sites are indicated.

Sperm samples were analyzed by electronic microscopy in order to reveal subtle morphological and ultrastructural defects that could not be visible through light microscopy and that could explain the in vitro hypofertility phenotype. Some abnormal sperm could be observed, while the acrosome seemed to be partially or completely detached from the nucleus. In some extreme cases, sperm could also look "inflated", showing an asymmetrical acrosome, completely untied from the nucleus, as exemplified in Figure 5a. A count of this particular phenotype revealed a greater abundance in KO (15\%) compared to WT $(3 \%)$ samples (Figure 5c). Some other sperm showed only one or the other defect, that were not considered in the previous quantitative evaluation. 

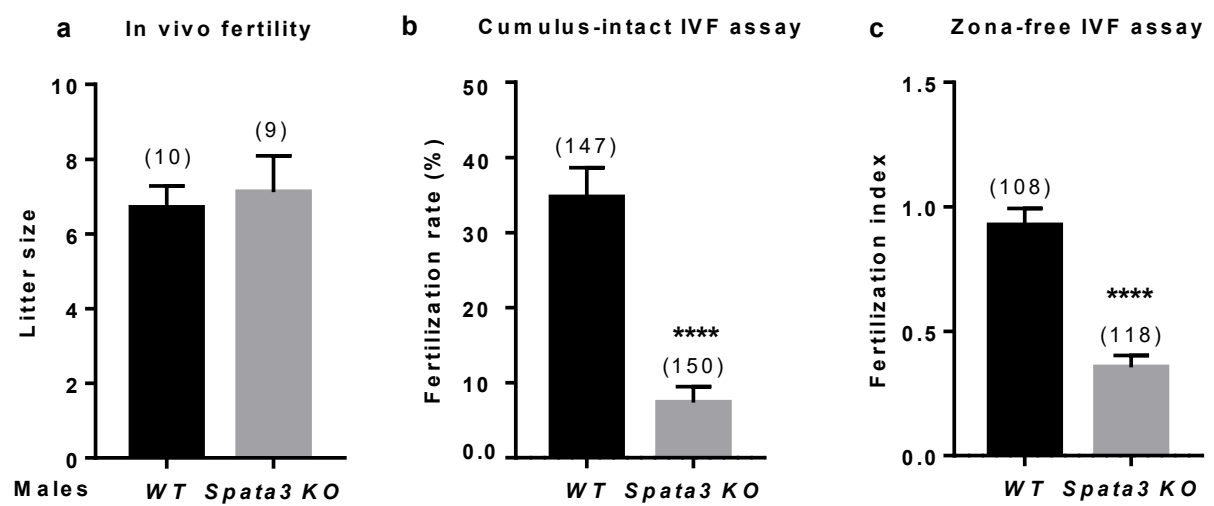

Figure 3. Evaluation of Spata3 KO male fertility. (a). Evaluation of in vivo fertility. WT females were mated with WT or $\mathrm{KO}$ males to evaluate their capacity to fertilize in vivo. After an overnight mating, vaginal plugs were checked. Litter size was counted after 3 weeks of gestation. Mean litter size \pm SEM. Numbers between brackets indicate the numbers of litters. (b). Evaluation of in vitro fertility. The fertilization rate (or mean percentage \pm SEM of fertilized eggs) was assessed by cumulus-intact IVF assay at $10^{6}$ (WT and KO) sperm per ml. It was decreased between oocytes inseminated by $\mathrm{KO}$ sperm compared to WT. Numbers between brackets indicate the numbers of oocytes. (c). Evaluation of in vitro fertility. The fertilization index (or mean \pm SEM of sperm number fused by egg) was assessed by zona-free IVF assay at $10^{5}$ (WT or KO) sperm per ml. It was decreased between oocytes inseminated by KO sperm compared to WT. Numbers between brackets indicate the numbers of oocytes. Experiments were repeated at least three times. ${ }^{* * * *} p<0.0001$.
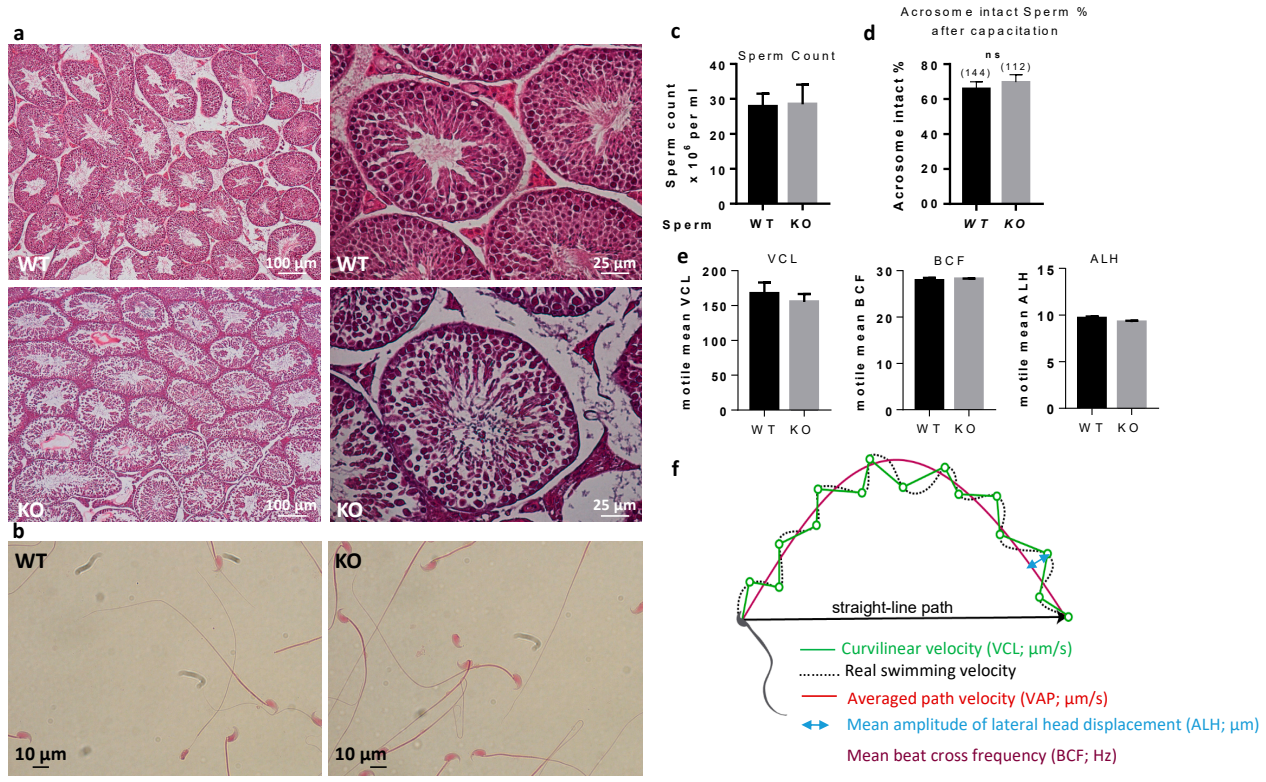

Figure 4. Histology of sperm and testes. (a). Adult testes were fixed in paraformaldehyde, sliced and colored with hematoxylin-eosin. (b). Papanicolaou staining of WT and KO sperm. (c). Sperm counts of WT and KO sperm. (d). Spontaneous acrosome reaction counting. There is no difference between WT and KO sperm in terms of acrosome reaction after capacitation. ns, not significant. (e). Analyses of sperm parameters by CASA. CASA analyzes of sperm movement parameters from Spata3 deleted mice showed no difference in comparison to those of WT sperm. As an example, here are presented: curvilinear velocity (VCL), beat/cross frequency (BCF) and amplitude of lateral head displacement (ALH). Data are represented as the mean \pm SEM of at least three sperm samples from males of each group. (f). Schematic representation of CASA measurements. 
a

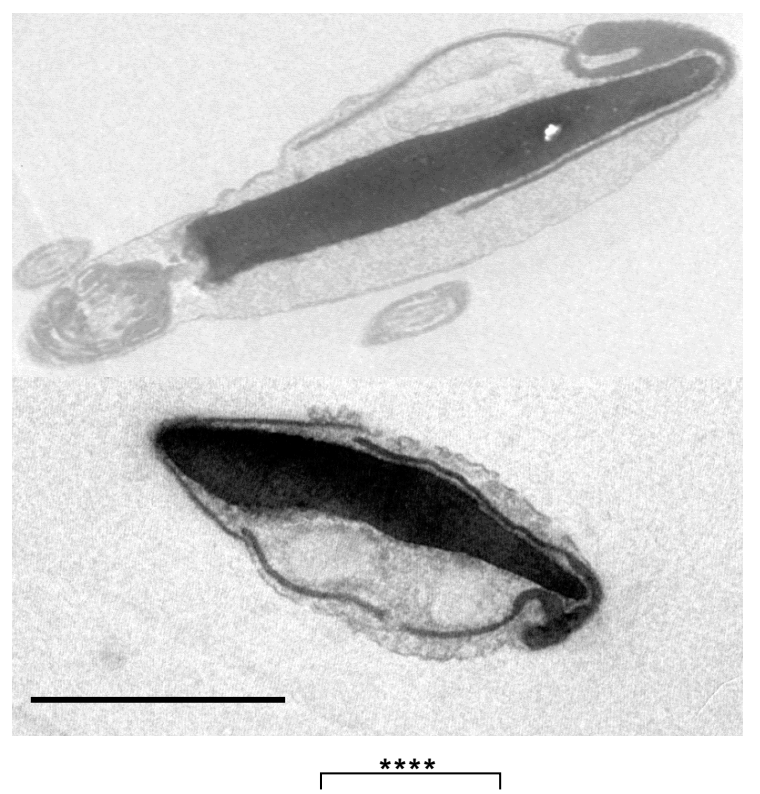

b

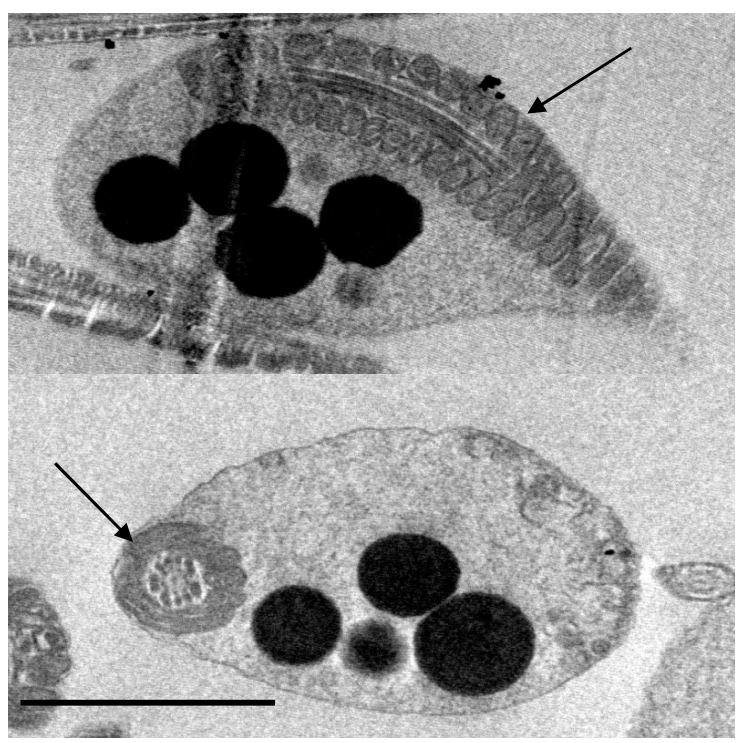

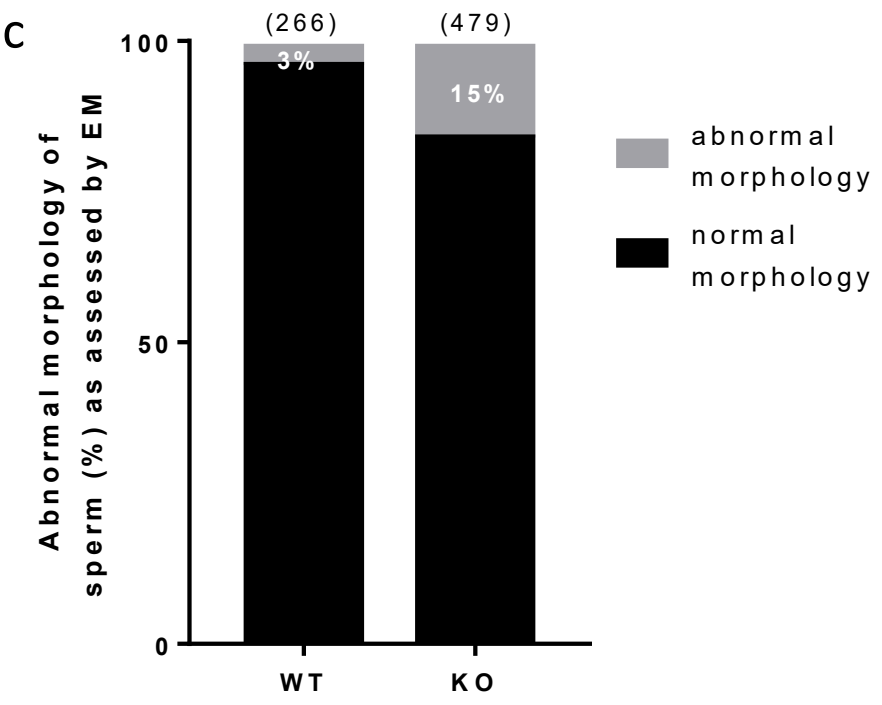

Figure 5. Electronic microscopy analysis of sperm. (a). Examples of abnormal sperm heads combining an "inflated" aspect, a detached and asymmetrical acrosome. (b). Examples of lipid droplets present in cytoplasmic bodies. The arrows indicate the presence of mitochondria within the flagellum. The scale bars represent $2 \mu \mathrm{m}$. (c). Counting of sperm showing the specific phenotype exemplified in panel a, among all observed spermatic heads, between WT and KO samples. Numbers between brackets indicate the numbers of sperm heads. ${ }^{* * *} p<0.0001$.

In addition, some dark droplets were visible, more frequently in $\mathrm{KO}$ compared to WT samples (Figure 5b). These droplets, suspected to contain lipids, are present within cytoplasmic remnants located around the midpiece of the flagellum, as attested to by the nearby presence of mitochondria.

In order to better characterize a putative metabolic defect resulting in such droplets, we used an Oil Red O staining on WT and KO sperm (Figure 6a). Up to 13.4\% and 9.4\% of KO sperm were positive for lipid droplets, before and after capacitation, respectively, whereas less than $0.5 \%$ of WT sperm presented staining in both conditions (Figure $6 \mathrm{~b}$ ). 


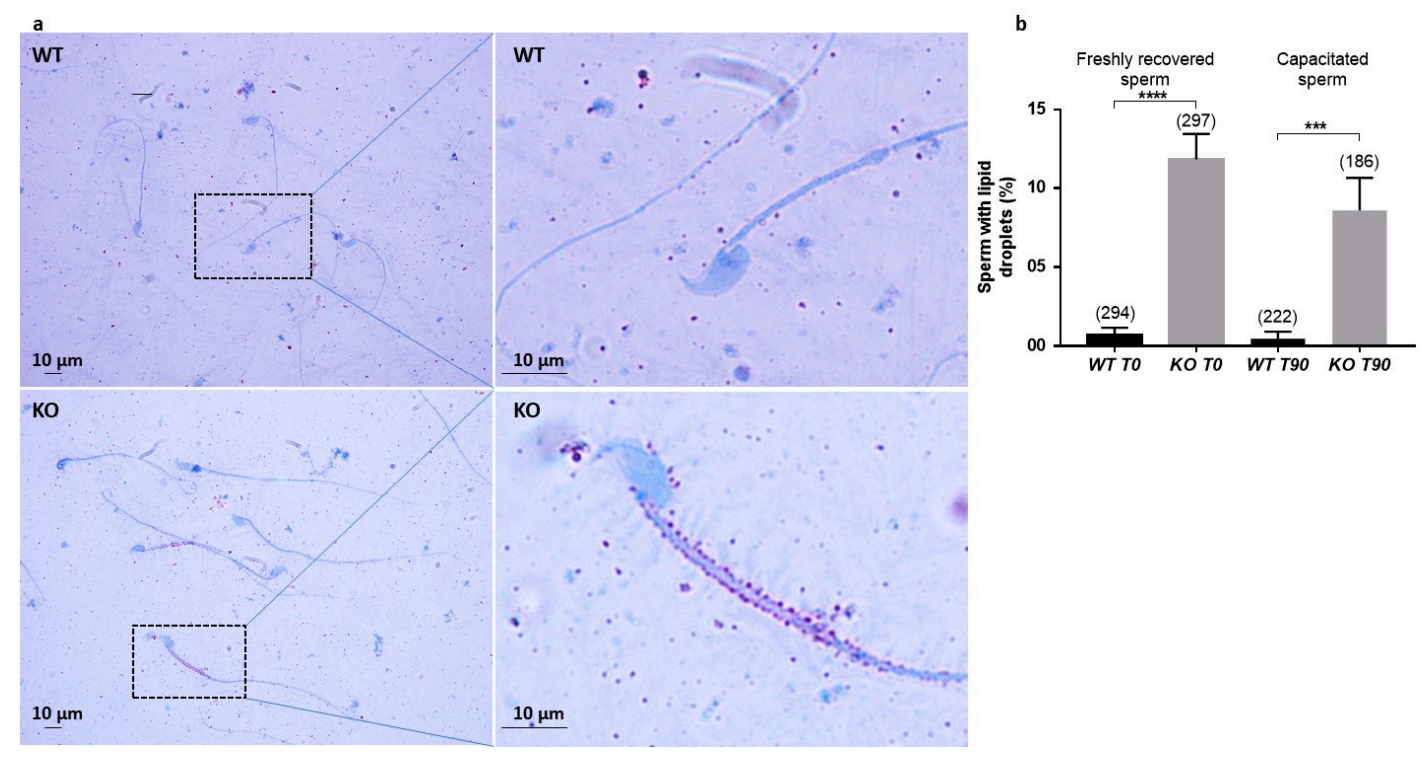

Figure 6. Lipid detection by Oil Red O staining. (a). Light microscopy detection of lipid droplets after Oil Red O staining. Right panels show higher magnification of regions framed in left panels. (b). Counting of sperm positive for lipid droplets after staining, before (T0) or after (T90) capacitation. Numbers between brackets indicate the counted sperm; ${ }^{* * *} p<0.001$ $* * * * p<0.0001$.

\section{Discussion}

In order to understand which gene within the Mafq1 QTL interval was responsible for the hypofertility phenotype observed in the Rc3 IRCS mice [7], we explored the list of candidate genes present in the interval. Spata3 matched all potential criterion of selection: the specific expression profile, regarding both the timing and location in differentiating sperm cells, the evolutionary conservation, the high number and predicted impact of missense changes between B6 and spretus SPATA3 proteins. The lack of information about its function left all hypotheses open. We therefore decided to generate a Spata3 null mouse to determine if the invalidation of the Spata3 gene could mimic the phenotype of the Rc3 IRCS mouse.

When this project was initiated, the reported structure of the Spata3 gene was the one reproduced in Figure 2a, as isoform 1, including 3 coding exons (Exons 1, 2 and 3 ) and additional variable $3^{\prime}$ UTR exons. A CRISPR/Cas9 strategy had been developed to target and affect exon 1 with RNA guides, therefore compromising the full ORF. Two independent $\mathrm{KO}$ lines were produced but they were found to be fully fertile in vivo (data not shown).

However, in 2018, databases revealed the existence of so far unsuspected isoforms of the gene. First, an additional isoform starts upstream of the reported translation start, at an ATG located 303 bp earlier, in phase ("exon 1 upstream", Figure 2a, isoform 2). Second, a new alternative isoform includes a new upstream first exon ("exon 1 bis", Figure 2a, isoform 3), followed by the classic coding exons 2 and 3, in phase. This new messenger therefore encodes a protein that shares the last 92 amino acids but completely differs from the initially known form at the $\mathrm{N}$-terminus and is not affected by the absence of the "classic" exon 1 . This gene organization is conserved, while isoforms 1 and 3 are present in mice and humans. In 2019, Zhou et al. reported new isoforms of the human SPATA3 gene [15]. They first identified a new isoform skipping exon 2, leading to a protein of 138 amino acids whose C-terminal end is different because of a frameshift, but whose abundance is unknown. They also described an alternative splicing within exon 1, where $27 \mathrm{bp}$ would be spliced away. However, this is rather a documented polymorphism, referenced as rs 72362780 , with different alleles of either 2 or 3 repeats of a 27 bp sequence encoding a QQPSPESTP motif, being almost equally frequent in the human population. 
The initial knock-out was therefore considered as a partial $\mathrm{KO}$, suppressing only some of the possible isoforms and probably leaving some not negligible residual SPATA3 activity. Another knock-out strategy was therefore adopted to delete all known coding exons. RNA guides located upstream exon 1 bis and downstream exon 3 were used to generate a complete gene deletion of about $8 \mathrm{~kb}$. Two independent $\mathrm{KO}$ lines were obtained for this complete invalidation that gave identical results. Sequencing of the locus confirmed the deletion of the coding exons and validated the precise breakpoints. The lack of expression of the Spata3 cDNA was confirmed by RT-PCR, in the absence of an efficient antibody to detect the SPATA3 protein by Western blot. When mated with WT females, homozygous KO males could sire normally and produce normal litters. The absence of Spata3 did not reproduce the in vivo hypofertility observed in Rc3 males [7]. However, in IVF assays, sperm lacking Spata3 expression were less efficient to fertilize WT oocytes. Both the fertilization rate of cumulus-intact oocytes and the fertilization index of zona-free oocytes were drastically reduced. This difference of phenotype between in vivo and in vitro conditions has already been observed. It is probably due to the differences between an optimal in vivo fertilization and the artificial context of IVF where some parameters are not ideally reproduced. Thus, IVF could exacerbate a defect that is compensated for under physiological conditions. This observation has already been encountered for the sperm specific Itgb1 KO [16] and for the mouse heterozygous for a Spaca6 deletion [17]. This reduction of IVF efficiency was also detected in Rc3 males.

No gross abnormality could be detected in the testicular histology neither in sperm counts, suggesting a normal spermatogenesis. The sperm movement parameters explored by CASA were not affected. A reduction of the motile fraction had been observed in Rc3 males but here the decrease in IVF yield cannot be explained by a reduced motility of sperm. Sperm ultrastructure was explored by electronic microscopy to observe more subtle sperm alterations, as were reported in the Rc3 IRCS model [7]. Some abnormal sperm could be observed, mainly affecting the acrosome. As observed in the Rc3 line, an acrosome detached from the nucleus is more frequently reported in KO than in WT sperm. The acrosome sometimes looks out of shape, far from the expected symmetric cap aspect. It is very likely that the totally abnormal appearance of the sperm shown in Figure 5a is not really the ejaculated sperm phenotype but rather the result of manipulations which further damage the structure of these KO sperm compared to WT sperm. It is anyway a sign of a particular fragility of $\mathrm{KO}$ sperm. In addition, dark droplets in persistent cytoplasmic remnants were also visible. An Oil Red $\mathrm{O}$ coloration was performed and confirmed the lipid nature of these droplets contents and that they are much more present in KO sperm. These cytoplasmic bodies should have been removed by Sertoli cells in the last steps of spermiogenesis. It is not clear if the phagocytosis process is altered in KO testes, but SPATA3 has been shown to be expressed in germ cells, not in Sertoli cells. Alternatively, a dysfunction of lipid metabolism could be present in KO sperm, leading to an excess of lipid droplets that fail to be correctly phagocyted by Sertoli cells. Why this process of lipids accumulation happens will need more analyses. It is difficult to estimate exactly what percentage of sperm is abnormal, as, more than $10 \%$ showed lipid droplets, $15 \%$ appeared fully disorganized at the acrosome level, and some others showed milder defects.

In conclusion, male mice lacking the expression of the Spata3 gene are fertile in vivo, thanks to the optimal context of sperm selection and gamete interaction. They are nevertheless hypofertile in vitro. Moreover, they have a higher frequency of abnormal sperm. Specifically, they have more abnormal acrosomes that tend to untie from the nucleus and more cytoplasmic bodies showing an imperfect spermiogenesis and spermiation.

The Spata3 KO, though modestly affecting sperm capacities, is therefore an incomplete reproduction of the Rc3 IRCS mouse line, that was considered as a model of partial globozoospermia. Two hypotheses can be proposed. It is possible that the complete suppression of SPATA3 protein expression in the invalidated mouse on one side and the expression of a modified SPATA3 protein in the IRCS on the other have different impacts on sperm function. The presence of a deleterious protein could be more damaging than 
its absence, if for example, some compensatory mechanisms can set up. Some of the polymorphisms existing between spretus and C57Bl/ 6 strains are indeed individually predicted to affect protein function. However, no prediction model can aggregate the effect of the 14 non-synonymous changes on the integrity of the different protein isoforms, especially on a protein of unknown function. The alternative hypothesis is that another gene within the Mafq1 interval contributes to the complex phenotype of the Rc3 males. One or more gene(s) present in the QTL interval and exhibiting variations in the spretus strain compared to the C57Bl/ 6 strain, likely missense changes affecting protein function but also possibly regulatory variants affecting protein levels, could also be dysfunctional in the Rc3 line. The cumulative effects of two or more disturbed genes could be necessary to affect more drastically sperm function, either directly if proteins are acting together or indirectly if they have different functions whose anomalies add at different stages or pathways to generate the defects profile at the end. A careful re-analysis of the identity and characteristics of the genes present in the Rc3 interval could suggest other candidates that could participate in the phenotype.

On the human side, SPATA3 and other potential candidate genes from the QTL region should be screened in infertile/hypofertile patients with signs reminiscent of globozoospermia. Furthermore, genes identified in this QTL region will be particularly closely analyzed in our future whole exome sequencing studies of infertile patients.

\section{Materials and Methods}

\subsection{Animals}

All animal experiments were performed in agreement with national guidelines for the care and use of laboratory animals. Experimentation was approved by the local ethics committee (C2EA-34, Comité d'éthique en matière d'expérimentation animale Paris Descartes) and the governmental ethical review committees via the APAFiS Application (Autorisation de projet utilisant des animaux à des fins scientifiques), under the registration reference APAFIS \#14124-2017072510448522 v26, A. Ziyyat (10/102018-10/10/2023).

Mice were purchased from Janvier Labs (Le Genest-Saint-Isle, France). All animals were maintained at the animal facility of the Cochin Institute (Paris) at a stable temperature $\left(21-23^{\circ} \mathrm{C}\right)$ and $14 \mathrm{~h}$ light $/ 10 \mathrm{~h}$ dark photoperiods, with free access to food and water.

\subsection{Generation of Transgenic Mice}

The Crispr/Cas9 approaches were performed at the Transgenesis and Homologous Recombination platform (PRHTEC) of the Cochin Institute, Paris, France. RNA guides were selected, produced by TACGENE (Muséum d'Histoire Naturelle, Paris, France) and tested in vitro (targeting upstream exon 1 bis: ACCGGACAGACATGGAACAG; GCTGGGACCCCTGTTAGCCAA; GTTGTGACATGCTCCAGGGAA- targeting downstream exon 3: GGACCCCAGGCCCACCGTC; GTAGAGAAGACTATCTCAAGG; GGCCCACCGTCCGGCAGGT).

They were preincubated with Cas9 and then microinjected into fertilized C57Bl/6J oocytes.

Embryos that had passed to the 2-cell stage were transferred into the oviducts of pseudopregnant females.

\subsection{PCR and RT-PCR}

Genotyping was performed on DNA extracted from tail biopsies (NucleoSpin ${ }^{\circledR}$ Tissue, Macherey-Nagel, Düren, Germany) using the GoTaq Flexi polymerase (Promega, Madison, WI, USA) under standard PCR conditions. Primers are listed in Table S2. The integrity of the areas targeted by the guides was tested to detect WT alleles. A large amplification encompassing the whole locus was also performed to detect deleted alleles.

RNA was obtained by TRIzol (Thermo Fischer Scientific, Waltham, MA, USA) extraction from WT and KO testes. Five micrograms of total RNA were treated with DNAse I (Promega) and retrotranscribed using the MMLV reverse transcriptase (Thermo Fis- 
cher Scientific) following manufacturer's instructions. RT-PCR was performed using the GoTaq Flexi polymerase to test the expression of Spata3 and a reference gene as normalization control.

\subsection{In Vivo and In Vitro Fertility Assessment}

Mice, aged from 8 to 12 weeks, were housed as one male and one female per cage. WT females were mated overnight with WT males or homozygous KO males and checked for vaginal plugs the next day. For each group, litter sizes were assessed.

Wild-type $5-8$ weeks-old female mice were superovulated with a $10 \mathrm{IU}$ pregnant mare serum gonadotrophin (PMSG) injection, then $48 \mathrm{~h}$ later with a $10 \mathrm{IU}$ human chorionic gonadotrophin (hCG) injection (Intervet, Beaucouzé, France). The next day, $14 \mathrm{~h}$ postinjection, animals were sacrificed by cervical dislocation. Cumuli oophori were retrieved by tearing the ampulla wall of the oviduct, placed in Ferticult medium (FertiPro, Beernem, Belgium) supplemented with $3 \%$ bovine serum albumin (BSA), and maintained at $37^{\circ} \mathrm{C}$ under $5 \% \mathrm{CO}_{2}$ in air, under mineral oil (Sigma Aldrich, Saint Quentin Fallavier, France).

For zona-free IVF assay, oocytes were released from the cumulus cells by 3-5 $\mathrm{min}$ incubation at $37^{\circ} \mathrm{C}$ with hyaluronidase (Sigma Aldrich) in M2 medium (Sigma Aldrich), rinsed and kept in Ferticult medium, BSA $3 \%$ at $37^{\circ} \mathrm{C}$ under a $5 \% \mathrm{CO}_{2}$ atmosphere with mineral oil (Sigma Aldrich). The zona pellucida was then dissolved with acidic Tyrode's (AT) solution ( $\mathrm{pH} 2.5$, Sigma Aldrich) under visual monitoring. Zona-free eggs were rapidly rinsed in a Ferticult medium, BSA $3 \%$ and kept at $37^{\circ} \mathrm{C}$ under a $5 \% \mathrm{CO}_{2}$ atmosphere for 2 to $3 \mathrm{~h}$ to recover.

Mouse spermatozoa were obtained from the cauda epididymis of control or $\mathrm{KO}$ male mice aged 8 to 13 -weeks. They were capacitated at $37{ }^{\circ} \mathrm{C}$ under a $5 \% \mathrm{CO}_{2}$ for $90 \mathrm{~min}$ in a $500 \mu \mathrm{L}$ drop of Ferticult medium supplemented with $3 \%$ BSA $(30 \mathrm{mg} / \mathrm{mL})$, under mineral oil.

Cumulus-intact or zona-free oocytes were inseminated in a $100 \mu \mathrm{L}$ drop of Ferticult medium, BSA $3 \%$ with capacitated spermatozoa at a final concentration of $1 \times 10^{6} / \mathrm{mL}$ or $1 \times 10^{5} / \mathrm{mL}$, respectively, for $3 \mathrm{~h}$. They were then washed and directly mounted in Vectashield/DAPI (Vector Laboratories, Burlingame, CA, USA) for observation under UV light (Zeiss Axioskop 20 microscope, Marly le Roi, France). Oocytes were considered fertilized when a fluorescent decondensed sperm head could be visualized within their cytoplasm.

\subsection{Histology, Staining and Immunostaining of Sperm and Testes}

Testes from adult WT and KO males were collected. They were fixed overnight in $4 \%(w / v)$ paraformaldehyde in PBS, dehydrated and embedded in paraffin. Serial $5 \mu \mathrm{m}$ thick sections were cut on a microtom and stored at room temperature. Testis sections were rehydrated before Hematoxylin-eosin (H\&E) staining.

For Immunofluorescence staining, slides were permeabilized during $10 \mathrm{~min}$ in PBS $1 \times$ and with triton $0.2 \%$ at RT, and were then saturated for $30 \mathrm{~min}$ in PBS, BSA $3 \%$ and Goat serum $10 \%$. Slides were incubated for $1 \mathrm{~h}$ in a polyclonal anti-TSARG1 antibody at 1:20 in PBS (T-18, sc-67901, Santa Cruz Biotechnology, Dallas, TX; reference no longer available), washed three times in PBS, BSA $1 \%$. DAPI $(5 \mu \mathrm{g} / \mathrm{mL}$, Sigma Aldrich) and secondary antibody (anti-Goat-Alexa 488 at $10 \mu \mathrm{g} / \mathrm{mL}$, ThermoFischer Scientific) were used at RT during $1 \mathrm{~h}$, followed by three washes and slides were mounted with the Dako Fluorescent mounting medium (Agilent, Santa Clara, CA, USA).

For Papanicolaou staining, sperm were retrieved from cauda epididymes in Ferticult medium and spread onto a Superfrost Plus slide (ThermoFischer Scientific). Sperm cells were fixed by incubation with PBS, paraformaldehyde $4 \%$ for $10 \mathrm{~min}$ and stained following the Papanicolaou protocol (Hematoxylin, OG6, EA50). Briefly, the slides were washed in 95\% ethanol and inserted in Harris hematoxylin for $3 \mathrm{~min}$ for nucleus counterstaining, and washed, stained with OG-6 dye (RAL diagnostics 05.12013, Martillac, France) and with EA-50 (RAL diagnostics 05.12019, Martillac, France), Then the slides were dehydrated (95\% ethanol absolute ethanol and xylene) and mounted with a permanent mounting medium. 
Freshly recovered or capacitated sperm were washed in PBS containing 1\% BSA, centrifuged at $300 \mathrm{~g}$ for $5 \mathrm{~min}$ and immediately fixed in $4 \%$ Paraformaldehyde (Electron Microscopy Sciences, PA, USA) in PBS-1\% BSA for $10 \mathrm{~min}$ at room temperature. In order to detect the sperm acrosomal status, after washing, fixed spermatozoa were stained with FITC-conjugated lectin Pisum Sativum Agglutinin (PSA; $25 \mu \mathrm{g} / \mathrm{mL}$ in PBS, Sigma Aldrich) for $15 \mathrm{~min}$. After repeated washing with PBS-BSA 1\%, a drop of sperm suspension was smeared on a slide, air-dried, mounted with Vectashield-DAPI. Detection was performed using a Nikon Eclipse E600 microscope Zeiss Axiophot epifluorescence microscope and images were digitally acquired with a camera (Coolpix 4500, Nikon, Champigny sur Marne, France).

For Oil Red O (ORO) staining, a drop of freshly recovered or capacitated sperm was smeared on a slide, air-dried and immediately fixed in PFA $4 \%$ and stored at $-80{ }^{\circ} \mathrm{C}$ until the day of use. Slides were post-fixed for $10 \mathrm{~min}$ in PFA $4 \%$, then rinsed and quenched with $\mathrm{NH}_{4} \mathrm{Cl} 50 \mathrm{mM}$ for $10 \mathrm{~min}$. After rinsing, cells were permeabilized for 4 min with triton X-100 0.5\%. Finally, cells were stained by an incubation of one hour with Oil-Red-O (Sigma Aldrich) and counter-stained with hematoxylin of Mayer. Lipids appeared as red droplets and nuclei appeared to be blue. The amount of lipid droplets by ORO staining in sperm cells was quantified using a light microscope.

\subsection{Mouse Sperm Motility Analysis}

Sperm motility was assessed by Computer Aided Sperm Analysis (CASA) using CEROS II apparatus (Hamilton Thorne, Beverly, MA, USA). Briefly, mouse spermatozoa were obtained from the cauda epididymis of Spata3 KO or WT C57BL6/J mice (8 to 10 weeks old) and capacitated at $37^{\circ} \mathrm{C}$ under $5 \% \mathrm{CO}_{2}$ for $90 \mathrm{~min}$ in a $500 \mu \mathrm{L}$ drop of Ferticult medium supplemented with $3 \%$ BSA, under mineral oil. The movements of at least 500 sperm cells per sample were analyzed in $20 \mu \mathrm{m}$ chambers (Leja Products B.V., NieuwVennep, Netherlands) with Zeiss AX10 Lab. A1 microscope (10× objective), using HT CASAII software.

The settings were as follows: acquisition rate, $60 \mathrm{~Hz}$; number of frames, 45 ; minimum head brightness, 175; minimum tail brightness, 80 ; minimum head size, $10 \mu \mathrm{m}^{2}$; minimum elongation gate, $1 \%$; maximum elongation gate, $100 \%$; objective magnification factor, 1.2.

The principal motility parameters measured were: curvilinear velocity (VCL), average path velocity (VAP), straight-line velocity (VSL), beat/cross frequency (BCF), amplitude of lateral head displacement (ALH).

\subsection{Electronic Microscopy}

Mouse spermatozoa from males were prepared as described above (IVF) and fixed by incubation in PBS supplemented with 2\% glutaraldehyde (Grade I, Sigma) for $2 \mathrm{~h}$ at room temperature. Samples were washed twice in PBS and post-fixed by incubation with $1 \%$ osmium tetroxide (Electron Microscopy Sciences), after which they were dehydrated by immersion in a graded series of alcohol solutions and embedded in Epon resin (Polysciences Inc.,Warrington, PA, USA). Ultra-thin sections $(90 \mathrm{~nm})$ were cut with a Reichert Ultracut $S$ ultramicrotome (Reichert-Jung AG, Wien, Austria) and were then stained with uranyl acetate and lead citrate. Sections were analyzed with a JEOL 1011 microscope and digital images were acquired with a Gatan Erlangshen CCD camera and Digital Micrograph software. The integrity of sperm organelles was checked including the acrosome, nucleus, flagellum, etc. Acrosomes with a space between the nuclear membrane and the inner acrosomal membrane were considered abnormal. Acrosomes with membranes in close contact were considered normal. The presence of residual bodies and lipid droplets was recorded.

\subsection{Statistical Analysis}

Results are expressed as mean \pm SEM of at least three independent experiments. For statistical analysis, $t$-Test was performed using GraphPad Prism version 7.00 for 
Windows, (GraphPad Software, La Jolla, CA, USA). Differences were considered statistically significant when $p<0.05$. When data were not normally distributed, a Welch's correction was applied.

Supplementary Materials: Supplementary materials can be found at https:/ /www.mdpi.com/1422 $-0067 / 22 / 4 / 1959 / \mathrm{s} 1$.

Author Contributions: Conceptualization, R.P., S.B. and A.Z.; methodology, M.-S.G., C.V., S.D., R.P., M.F., L.S. and C.I.-R.; validation, M.-S.G., C.V., S.D. and C.I.-R.; formal analysis, A.Z. and S.B.; investigation, M.-S.G., C.V., S.D., L.S. and C.I.-R.; writing—original draft preparation, S.B. and A.Z.; writing-review and editing, A.Z. and S.B.; visualization, A.Z., and S.B.; supervision, S.B. and A.Z.; funding acquisition, A.Z. All authors have read and agreed to the published version of the manuscript.

Funding: This research received no external funding. This work was supported by the Institut National de la Santé et de la Recherche Médicale (INSERM), the Centre National de la Recherche Scientifique (CNRS), the Université de Paris.

Institutional Review Board Statement: All animal experiments were performed in agreement with national guidelines for the care and use of laboratory animals. The study was conducted according to the guidelines of the Declaration of Helsinki and was approved by the local ethics committee. (C2EA34, Comité d'éthique en matière d'expérimentation animale Paris Descartes) and the governmental ethical review committees via the APAFiS Application (Autorisation de projet utilisant des animaux à des fins scientifiques), under the registration reference APAFIS \#14124-2017072510448522 v26, A. Ziyyat (10/10/2018-10/10/2023).

Informed Consent Statement: Not applicable.

Data Availability Statement: Not applicable.

Acknowledgments: The authors thank the staff of the animal facility and the PRHTEC platform of the Cochin Institute, and A. Schmitt from the Electronic Microscopy Facility (Cochin Institute, Paris). The authors thank A. Touré and F. Saez for providing advice.

Conflicts of Interest: The authors declare no conflict of interest.

\begin{tabular}{ll}
\multicolumn{2}{l}{ Abbreviations } \\
WT & Wild-type \\
KO & Knock-out \\
IRCS & Interspecific Recombinant Congenic Strain \\
FR & Fertilization Rate \\
FI & Fertilization Index \\
IVF & In Vitro Fertilization \\
CASA & Computer Assisted Sperm Analysis \\
QTL & Quantitative Trait Locus \\
DAPI & $4^{\prime}, 6$-diamidino-2-phenylindole
\end{tabular}

\section{References}

1. Schultz, N.; Hamra, F.K.; Garbers, D.L. A multitude of genes expressed solely in meiotic or postmeiotic spermatogenic cells offers a myriad of contraceptive targets. Proc. Natl. Acad. Sci. USA 2003, 100, 12201-12206. [CrossRef] [PubMed]

2. Celse, T.; Cazin, C.; Mietton, F.; Martinez, G.; Martinez, D.; Thierry-Mieg, N.; Septier, A.; Guillemain, C.; Beurois, J.; Clergeau, A.; et al. Genetic analyses of a large cohort of infertile patients with globozoospermia, DPY19L2 still the main actor, GGN confirmed as a guest player. Hum. Genet. 2020. [CrossRef] [PubMed]

3. Coutton, C.; Abada, F.; Karaouzene, T.; Sanlaville, D.; Satre, V.; Lunardi, J.; Jouk, P.S.; Arnoult, C.; Thierry-Mieg, N.; Ray, P.F. Fine characterisation of a recombination hotspot at the DPY19L2 locus and resolution of the paradoxical excess of duplications over deletions in the general population. PLoS Genet. 2013, 9, e1003363. [CrossRef] [PubMed]

4. Dam, A.H.; Koscinski, I.; Kremer, J.A.; Moutou, C.; Jaeger, A.S.; Oudakker, A.R.; Tournaye, H.; Charlet, N.; Lagier-Tourenne, C.; van Bokhoven, H.; et al. Homozygous mutation in SPATA16 is associated with male infertility in human globozoospermia. Am. J. Hum. Genet. 2007, 81, 813-820. [CrossRef] [PubMed] 
5. Liu, G.; Shi, Q.W.; Lu, G.X. A newly discovered mutation in PICK1 in a human with globozoospermia. Asian J. Androl. 2010, 12, 556-560. [CrossRef] [PubMed]

6. Yatsenko, A.N.; O’Neil, D.S.; Roy, A.; Arias-Mendoza, P.A.; Chen, R.; Murthy, L.J.; Lamb, D.J.; Matzuk, M.M. Association of mutations in the zona pellucida binding protein 1 (ZPBP1) gene with abnormal sperm head morphology in infertile men. Mol. Hum. Reprod. 2012, 18, 14-21. [CrossRef] [PubMed]

7. Vatin, M.; Girault, M.S.; Firlej, V.; Marchiol, C.; Ialy-Radio, C.; Montagutelli, X.; Vaiman, D.; Barbaux, S.; Ziyyat, A. Identification of a New QTL Region on Mouse Chromosome 1 Responsible for Male Hypofertility: Phenotype Characterization and Candidate Genes. Int. J. Mol. Sci. 2020, 21, 8506. [CrossRef]

8. Fu, J.J.; Lu, G.X.; Li, L.Y.; Liu, G.; Xing, X.W.; Liu, S.F. Molecular cloning for testis spermatogenesis cell apoptosis related gene TSARG1 and Mtsarg1 and expression analysis for Mtsarg1 gene. Yi Chuan Xue Bao 2003, 30, $25-29$.

9. Li, L.; Liu, G.; Fu, J.J.; Li, L.Y.; Tan, X.J.; Yang, S.; Lu, G.X. Molecular cloning and characterization of a novel transcript variant of Mtsarg1 gene. Mol. Biol. Rep. 2009, 36, 1023-1032. [CrossRef] [PubMed]

10. Malcher, A.; Rozwadowska, N.; Stokowy, T.; Kolanowski, T.; Jedrzejczak, P.; Zietkowiak, W.; Kurpisz, M. Potential biomarkers of nonobstructive azoospermia identified in microarray gene expression analysis. Fertil. Steril. 2013, 100, 1686-1694.e7. [CrossRef] [PubMed]

11. Oh, J.J.; Shivakumar, M.; Miller, J.; Verma, S.; Lee, H.; Hong, S.K.; Lee, S.E.; Lee, Y.; Lee, S.J.; Sung, J.; et al. An exome-wide rare variant analysis of Korean men identifies three novel genes predisposing to prostate cancer. Sci. Rep. 2019, 9, 17173. [CrossRef] [PubMed]

12. Darde, T.A.; Lecluze, E.; Lardenois, A.; Stevant, I.; Alary, N.; Tuttelmann, F.; Collin, O.; Nef, S.; Jegou, B.; Rolland, A.D.; et al. The ReproGenomics Viewer: A multi-omics and cross-species resource compatible with single-cell studies for the reproductive science community. Bioinformatics 2019, 35, 3133-3139. [CrossRef] [PubMed]

13. Darde, T.A.; Sallou, O.; Becker, E.; Evrard, B.; Monjeaud, C.; Le Bras, Y.; Jegou, B.; Collin, O.; Rolland, A.D.; Chalmel, F. The ReproGenomics Viewer: An integrative cross-species toolbox for the reproductive science community. Nucleic Acids Res. 2015, 43, W109-W116. [CrossRef] [PubMed]

14. Okada, Y.; Scott, G.; Ray, M.K.; Mishina, Y.; Zhang, Y. Histone demethylase JHDM2A is critical for Tnp1 and Prm1 transcription and spermatogenesis. Nature 2007, 450, 119-123. [CrossRef] [PubMed]

15. Zhou, B.; Wei, C.; Khan, M.A.; Chen, H.; Fu, J. Characterization and molecular cloning of novel isoforms of human spermatogenesis associated gene SPATA3. Mol. Biol. Rep. 2019, 46, 3827-3834. [CrossRef] [PubMed]

16. Barraud-Lange, V.; Ialy-Radio, C.; Chalas, C.; Holtzmann, I.; Wolf, J.P.; Barbaux, S.; Ziyyat, A. Partial Sperm beta1 Integrin Subunit Deletion Proves its Involvement in Mouse Gamete Adhesion/Fusion. Int. J. Mol. Sci. 2020, 21, 8494. [CrossRef] [PubMed]

17. Barbaux, S.; Ialy-Radio, C.; Chalbi, M.; Dybal, E.; Homps-Legrand, M.; Do Cruzeiro, M.; Vaiman, D.; Wolf, J.P.; Ziyyat, A. Sperm SPACA6 protein is required for mammalian Sperm-Egg Adhesion/Fusion. Sci. Rep. 2020, 10, 5335. [CrossRef] [PubMed] 